\title{
TEICHMÜLLER SPACES AND BOUNDED SYMMETRIC DOMAINS DO NOT MIX ISOMETRICALLY
}

\author{
Stergios M. Antonakoudis
}

\begin{abstract}
This paper shows that, in dimensions two or more, there are no holomorphic isometries between Teichmüller spaces and bounded symmetric domains in their intrinsic Kobayashi metric.
\end{abstract}

\section{Contents}

1 Introduction . . . . . . . . . . . . . . . . . . . . . 453

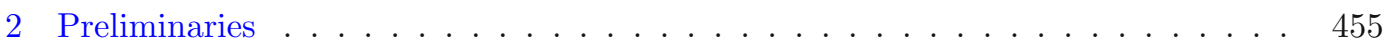

3 Extremal length geometry . . . . . . . . . . . . . . . . . . . . . . . 457

4 Symmetric spaces versus Teichmüller spaces . . . . . . . . . . . . . . . . . . . . 461

References . . . . . . . . . . . . . . . . . . . . . 464

\section{Introduction}

We study holomorphic maps between Teichmüller spaces $\mathcal{T}_{g, n} \subset \mathbb{C}^{3 g-3+n}$ and bounded symmetric domains $\mathcal{B} \subset \mathbb{C}^{N}$ in their intrinsic Kobayashi metric. The main result in this paper is the following theorem.

Theorem 1.1. Let $\mathcal{B}$ be a bounded symmetric domain and $\mathcal{T}_{g, n}$ be a Teichmüller space with $\operatorname{dim}_{\mathbb{C}} \mathcal{B}, \operatorname{dim}_{\mathbb{C}} \mathcal{T}_{g, n} \geq 2$. There are no holomorphic isometric immersions

$$
\mathcal{B} \stackrel{f}{\longleftrightarrow} \mathcal{T}_{g, n} \quad \text { or } \quad \mathcal{T}_{g, n} \stackrel{f}{\longrightarrow} \mathcal{B}
$$

equivalently, there are no holomorphic maps $f$ such that $d f$ is an isometry for the Kobayashi norms on tangent spaces.

The proof involves ideas from geometric topology and leverages the description of Teichmüller geodesics in terms of measured foliations and extremal length on Riemann surfaces.

We note the following immediate corollary.

COROLlary 1.2. There is no locally symmetric variety $\mathcal{V}$ isometrically immersed in the moduli space of curves $\mathcal{M}_{g, n}$, nor is there an isometric copy of $\mathcal{M}_{g, n}$ in $\mathcal{V}$, for the Kobayashi metrics, so long as both have dimension two or more. 
A feature that Teichmüller spaces and bounded symmetric domains have in common is that they contain holomorphic isometric copies of $\mathbb{C H}^{1}$ through every point and complex direction; in particular, in complex dimension one, Teichmüller spaces and bounded symmetric domains coincide.

In higher dimensions, it is known [FM12, see discussion \$13.2.1] that there are many holomorphic isometries between Teichmüller spaces $f: \mathcal{T}_{g, n} \hookrightarrow \mathcal{T}_{h, m}$ and bounded symmetric domains $f: \mathcal{B} \hookrightarrow \widetilde{\mathcal{B}}$, respectively, in their intrinsic Kobayashi metric.

Informally, our results show that in dimensions two or more Teichmüller spaces and bounded symmetric domains do not mix isometrically.

As an application of Theorem 1.1, we prove:

Theorem 1.3. Let $(\mathcal{M}, g)$ be a complete Kähler manifold with $\operatorname{dim}_{\mathbb{C}} \mathcal{M} \geq 2$ and holomorphic sectional curvature at least -4 . There is no holomorphic map $f: \mathcal{M} \rightarrow$ $\mathcal{T}_{g, n}$ such that $d f$ is an isometry on tangent spaces.

Proof. The monotonicity of holomorphic sectional curvature under holomorphic maps and the existence of (totally geodesic) holomorphic isometries $\mathbb{C H}^{1} \hookrightarrow \mathcal{T}_{g, n}$ through every complex direction imply that $\mathcal{M}$ has constant holomorphic curvature -4. [Roy71] Since $\mathcal{M}$ is a complete Kähler manifold, we have $\mathcal{M} \cong \mathbb{C H}^{N}$, which is impossible when $N \geq 2$ by Theorem 1.1 .

The following corollary is immediate.

COROLlary 1.4. There is no holomorphic, totally geodesic isometry from a Kähler manifold $\mathcal{M}$ into a Teichmüller space $\mathcal{T}_{g, n}$, so long as $\mathcal{M}$ has dimension two or more.

Questions. We conclude with two open questions.

1. Is there a holomorphic map $f:(\mathcal{M}, g) \rightarrow \mathcal{T}_{g, n}$ from a Hermitian manifold with $\operatorname{dim}_{\mathbb{C}} \mathcal{M} \geq 2$ such that $d f$ is an isometry on tangent spaces?

2. Is there a round complex two-dimensional linear slice in $T_{X} \mathcal{T}_{g, n}$ ?

Theorems 1.1 and 1.3 suggest that the answers to both questions are negative.

Notes and References. For an introduction to Teichmüller spaces, we refer to [GL00] and [Hub06]; for an introduction to symmetric spaces and their intrinsic Kobayashi metric, we refer to [Hel78], [Sat80] and [Kob98], respectively. We note that the Kobayashi metric of a bounded symmetric domain $\mathcal{B}$ does not coincide with its Hermitian symmetric metric, unless it has rank one ie. $\mathcal{B} \cong \mathbb{C H}^{N}$.

In his pioneering paper [Roy71], H. L. Royden showed that the Kobayashi metric of $\mathcal{T}_{g, n}$ coincides with its classical Teichmüller metric and, using this result, he proved that, when $\operatorname{dim}_{\mathbb{C}} \mathcal{T}_{g} \geq 2$, the group of holomorphic automorphisms $\operatorname{Aut}\left(\mathcal{T}_{g}\right)$ is discrete; hence, in particular, $\mathcal{T}_{g, n}$ is not a symmetric domain. A proof that $\operatorname{Aut}\left(\mathcal{T}_{g, n}\right)$ is discrete for all finite-dimensional Teichmüller spaces of dimension two or more is given in [EM03]. 
The existence of isometrically immersed curves, known as Teichmüller curves, in $\mathcal{M}_{g, n}$ has far-reaching applications in the dynfamics of billiards in rational polygons. [Vee89], [McM03] Corollary 1.2 shows that there are no higher dimensional, locally symmetric, analogues of Teichmüller curves.

In a follow-up paper [Ant], we use the results from this work to show that Theorem 1.1 is true for convex domains $\mathcal{B} \subset \mathbb{C}^{N}$ as well. Finally, we refer to [Ant], where a similar result to Theorem 1.1 is proved for isometric submersions.

\section{Preliminaries}

Let $\mathcal{T}_{g, n}$ denote the Teichmüller space of marked Riemann surfaces of genus $g$ with $n$ punctures; it is the orbifold universal cover of the moduli space of curves $\mathcal{M}_{g, n}$ and it is naturally a complex manifold of dimension $3 g-3+n$. It is known [Ber81] that Teichmüller space can be realized as a (contractible) bounded domain $\mathcal{T}_{g, n} \subset$ $\mathbb{C}^{3 g-3+n}$, by the Bers embeddings.

Let $\mathcal{B} \subset \mathbb{C}^{N}$ be a bounded domain; we call $\mathcal{B}$ a bounded symmetric domain if every point $p \in \mathcal{B}$ is an isolated fixed point of a holomorphic involution $\sigma_{p}: \mathcal{B} \rightarrow \mathcal{B}$, with $\sigma_{p}^{2}=\operatorname{id}_{\mathcal{B}}$. Bounded symmetric domains are contractible and homogeneous as complex manifolds. It is classically known [Hel78] that all Hermitian symmetric spaces of non-compact type can be realized as bounded symmetric domains $\mathcal{B} \subset \mathbb{C}^{N}$, by the Harish-Chandra embeddings.

The unit disk $\Delta \cong\{z \in \mathbb{C}:|z|<1\}$ is a bounded symmetric domain; in fact, it is the unique (up to isomorphism) contractible bounded domain of complex dimension one. We denote by $\mathbb{C} \mathbb{H}^{1}$ the unit disk equipped with its Poincaré metric $|d z| /\left(1-|z|^{2}\right)$ of constant curvature -4 , which we will refer to as the complex hyperbolic line. Schwarz lemma shows that every holomorphic map $f: \mathbb{C H}^{1} \rightarrow \mathbb{C H}^{1}$ is non-expanding.

The Kobayashi metric [Kob98]. Let $\mathcal{B} \subset \mathbb{C}^{N}$ be a bounded domain, its intrinsic Kobayashi metric is the largest complex Finsler metric such that every holomorphic map $f: \mathbb{C H}^{1} \rightarrow \mathcal{B}$ is non-expanding: $\|d f\|_{\mathcal{B}} \leq 1$. It determines both a family of norms $\|\cdot\|_{\mathcal{B}}$ on the tangent bundle $T \mathcal{B}$ and a distance $d_{\mathcal{B}}(\cdot, \cdot)$ on pairs of points.

The Kobayashi metric has the fundamental property that every holomorphic map between complex domains is non-expanding; in particular, every holomorphic automorphism is an isometry. The Kobayashi metric of complex domain depends only on its structure as a complex manifold.

\section{Examples.}

(1) $\mathbb{C} \mathbb{H}^{1}$ realises the unit disk $\Delta$ with its Kobayashi metric. The Kobayashi metric on the unit ball $\mathbb{C H}^{2} \cong\left\{\left.(z, w)|| z\right|^{2}+|w|^{2}<1\right\} \subset \mathbb{C}^{2}$ coincides with its unique (complete) invariant Kaëhler metric of constant holomorphic curvature -4.See [Kob98, Example 3.1.24]. 
(2) The Kobayashi metric on the bi-disk $\mathbb{C} \mathbb{H}^{1} \times \mathbb{C H}^{1}$ coincides with the sup-metric of the two factors. It is a complex Finsler metric; it is not a Hermitian metric. [Kob98, Corollary 3.1.10].

(3) The Kobayashi metric on $\mathcal{T}_{g, n}$ coincides with the classical Teichmüller metric, which endows $\mathcal{T}_{g, n}$ with the structure of a complete geodesic metric space. [Roy71]

Incidentally, Examples $1 \& 2$ describe all bounded symmetric domains up to isomorphism, in dimension one and two. We discuss Example 3 in more detail below.

Teichmüller space [GL00], [Hub06]. Let $\Sigma_{g, n}$ be a connected, oriented surface of genus $g$ and $n$ punctures and $\mathcal{T}_{g, n}$ denote the Teichmüller space of Riemann surfaces marked by $\Sigma_{g, n}$. A point in $\mathcal{T}_{g, n}$ is specified by an orientation preserving homeorphism $\phi: \Sigma_{g, n} \rightarrow X$ to a Riemann surface of finite type, up to a natural equivalence relation ${ }^{1}$.

Teichmüller space $\mathcal{T}_{g, n}$ is naturally a complex manifold of dimension $3 g-3+n$ and forgetting the marking realises $\mathcal{T}_{g, n}$ as the complex orbifold universal cover of the moduli space $\mathcal{M}_{g, n}$. When it is clear from the context we often denote a point specified by $\phi: \Sigma_{g, n} \rightarrow X$ simply by $X$.

Quadratic differentials. For each $X \in \mathcal{T}_{g, n}$, we let $Q(X)$ denote the space of holomorphic quadratic differentials $q=q(z)(d z)^{2}$ on $X$ with finite total mass: $\|q\|_{1}=$ $\int_{X}|q(z)||d z|^{2}<+\infty$, which means that $q$ has at worse simple poles at the punctures of $X$.

The tangent and cotangent spaces to Teichmüller space at $X \in \mathcal{T}_{g, n}$ are described in terms of the natural pairing $(q, \mu) \mapsto \int_{X} q \mu$ between the space $Q(X)$ and the space $M(X)$ of $L^{\infty}$-measurable Beltrami differentials on $X$; in particular, the tangent $T_{X} \mathcal{T}_{g, n}$ and cotangent $T_{X}^{*} \mathcal{I}_{g, n}$ spaces are naturally isomorphic to $M(X) / Q(X)^{\perp}$ and $Q(X)$, respectively.

The Teichmüller-Kobayashi metric on $\mathcal{T}_{g, n}$ is given by norm duality on the tangent space $T_{X} \mathcal{T}_{g, n}$ from the norm $\|q\|_{1}=\int_{X}|q|$ on the cotangent space $Q(X)$ at $X$. The corresponding distance function is given by the formula $d_{\mathcal{T}_{g, n}}(X, Y)=$ inf $\frac{1}{2} \log K(\phi)$ and measures the minimal dilatation $K(\phi)$ of a quasiconformal map $\phi: X \rightarrow Y$ respecting their markings.

We denote by $Q \mathcal{T}_{g, n} \cong T^{*} \mathcal{T}_{g, n}$ the complex vector-bundle of holomorphic quadratic differentials over $\mathcal{T}_{g, n}$ and by $Q_{1} \mathcal{T}_{g, n}$ the associated sphere-bundle of quadratic differentials with unit mass. There is a natural norm-preserving action of $\mathrm{PSL}_{2}(\mathbb{R})$ on $Q \mathcal{T}_{g, n}$, with the diagonal matrices giving the geodesic flow for the TeichmüllerKobayashi metric of $\mathcal{T}_{g, n}$. For each $(X, q) \in Q_{1} \mathcal{T}_{g, n}$, the orbit $\operatorname{PSL}_{2}(\mathbb{R}) \cdot(X, q) \subset$ $Q_{1} \mathcal{I}_{g, n}$ induces a holomorphic totally-geodesic isometry $\mathbb{C H}^{1} \cong \mathrm{SO}_{2}(\mathbb{R}) \backslash \mathrm{PSL}_{2}(\mathbb{R}) \hookrightarrow$ $\mathcal{T}_{g, n}$, which we refer to as the Teichmüller disk generated by $(X, q)$.

\footnotetext{
1 Two marked Riemann surfaces $\phi: \Sigma_{g, n} \rightarrow X, \psi: \Sigma_{g, n} \rightarrow Y$ are equivalent if $\psi \circ \phi^{-1}: X \rightarrow Y$ is isotopic to a holomorphic bijection.
} 
Measured foliations [FLP79]. Let $\mathcal{M F}_{g, n}$ denote the space of equivalent classes of nonzero (singular) measured foliations on $\Sigma_{g, n}$, where two measured foliations $\mathcal{F}, \mathcal{G}$ are equivalent $\mathcal{F} \sim \mathcal{G}$ if they differ by a finite sequence of Whitehead moves followed by an isotopy of $\Sigma_{g, n}$ preserving their transverse measures. It is known that $\mathcal{M} \mathcal{F}_{g, n}$ has the structure of a piecewise linear manifold, which is homeomorphic to $\mathbb{R}^{6 g-6+2 n} \backslash\{0\}$.

The geometric intersection number of a pair of measured foliations $\mathcal{F}, \mathcal{G}$, denoted by $i(\mathcal{F}, \mathcal{G})$, induces a continuous map $i(\cdot, \cdot): \mathcal{M F}_{g, n} \times \mathcal{M F}_{g, n} \rightarrow \mathbb{R}_{\geq 0}$, which extends the geometric intersection pairing on the space of (isotopy classes of) simple closed curves on $\Sigma_{g, n}$. [Bon88]

Given $\mathcal{F} \in \mathcal{M F}_{g, n}$ and $X \in \mathcal{T}_{g, n}$, we let $\lambda(\mathcal{F}, X)$ denote the extremal length of $\mathcal{F}$ on the Riemann surface $X$ given by the formula $\lambda(\mathcal{F}, X)=\sup \frac{\ell_{\rho}(\mathcal{F})^{2}}{\operatorname{area}(\rho)}$, where $\ell_{\rho}(\mathcal{F})$ denotes the $\rho$-length of $\mathcal{F}$ and the supremum is over all (Borel-measurable) conformal metrics $\rho$ of finite area on $X$.

Each nonzero quadratic differential $q \in Q(X)$ induces a conformal metric $|q|$ on $X$, which is non-singular of zero curvature away from the zeros of $q$, and a measured foliation $\mathcal{F}(q)$ tangent to vectors $v=v(z) \frac{\partial}{\partial z}$ with $q(v)=q(z)(v(z))^{2}<0$. The transverse measure of the foliation $\mathcal{F}(q)$ is (locally) given by integrating $|\operatorname{Re}(\sqrt{q})|$ along arcs transverse to its leaves.

We refer to $\mathcal{F}(q)$ as the vertical measured foliation induced from $(X, q)$. In local coordinates, where $q=d z^{2}$ (such coordinates exist away from the zeros of $q$ ), the metric $|q|$ coincides with the Euclidean metric $|d z|$ in the plane and the measured foliation $\mathcal{F}(q)$ has leaves given by vertical lines and transverse measure by the total horizontal variation $|\operatorname{Re}(d z)|$. We note that the measured foliation $\mathcal{F}(-q)$ has (horizontal) leaves orthogonal to $\mathcal{F}(q)$ and the product of their transverse measures is just the area form of the conformal metric $|q|$ induced from $q$.

When it is clear from the context we often identify the measured foliation $\mathcal{F}(q)$ with its equivalence class in $\mathcal{M F}_{g, n}$. The following fundamental theorem will be used in the next section.

Theorem 2.1 ([HM79]; Hubbard-Masur). Let $X \in \mathcal{T}_{g, n}$; the map $q \mapsto \mathcal{F}(q)$ induces a homeomorphism $Q(X) \backslash\{0\} \cong \mathcal{M F}_{g, n}$. Moreover, $|q|$ is the unique extremal metric for $\mathcal{F}(q)$ on $X$ and its extremal length is given by the formula $\lambda(\mathcal{F}, X)=\|q\|_{1}$.

\section{Extremal length geometry}

Let $\mathbb{C} \mathbb{H}^{2} \cong\left\{\left.(z, w) \quad|\quad| z\right|^{2}+|w|^{2}<1\right\} \subset \mathbb{C}^{2}$ denote the complex hyperbolic plane, realized as the round unit ball with its Kobayashi metric. In this section we will use measured foliations and extremal length on Riemann surfaces to prove:

Theorem 3.1. There is no holomorphic isometry $f: \mathbb{C H}^{2} \hookrightarrow \mathcal{T}_{g, n}$ for the Kobayashi metric. 
Outline of the proof. The proof leverages the fact that extremal length provides a link between the geometry of Teichmüller geodesics and the geometric intersection pairing for measured foliations.

Firstly, we note that such an isometry $f$ would be totally-geodesic, it would send real geodesics in $\mathbb{C H}^{2}$ to Teichmüller geodesics in $\mathcal{T}_{g, n}$, preserving their length. See [EKK94, Theorem 5] for a proof using a deep result due to Slodkowski [Sło91]. By Theorem 2.1, we can parametrize the set of Teichmüller geodesic rays from any base point $X \in \mathcal{T}_{g, n}$ by the subspace of measured foliations $\mathcal{F} \in \mathcal{M} \mathcal{F}_{g, n}$ with extremal length $\lambda(\mathcal{F}, X)=1$.

Assuming the existence of $f$, we consider pairs of measured foliations that parametrize orthogonal geodesic rays in the image of a totally real geodesic hyperbolic plane $\mathbb{R H}^{2} \subset \mathbb{C H}^{2}$. We obtain a contradiction by computing their geometric intersection number in two different ways.

On the one hand, we use the geometry of complex hyperbolic horocycles and extremal length to show that the geometric intersection number does not depend on the choice of the totally real geodesic plane. On the other hand, by a direct geometric argument we show that this is impossible. More precisely, we have:

Proposition 3.2. Let $q \in Q_{1} \mathcal{T}_{g, n}$ and $\mathcal{G} \in \mathcal{M F}_{g, n}$. There exist $v_{1}, \ldots, v_{N} \in \mathbb{C}^{*}$ such that $i\left(\mathcal{F}\left(\mathrm{e}^{i \theta} q\right), \mathcal{G}\right)=\sum_{i=1}^{N}\left|\operatorname{Re}\left(\mathrm{e}^{i \theta / 2} v_{i}\right)\right|$ for all $\theta \in \mathbb{R} / 2 \pi \mathbb{Z}$.

The proof of the proposition is given at the end of the section.

See $\S 2$ for background material in Teichmüller theory and notation.

Complex hyperbolic horocycles. Let $\gamma:[0, \infty) \rightarrow \mathbb{C H}^{2}$ be a geodesic ray with unit speed. Since $\mathbb{C H}^{2}$ is a homogeneous space, we have $\gamma=\alpha \circ \gamma_{1}$, where $\gamma_{1}(t)=$ $(\tanh (t), 0)$, for $t \geq 0$, and $\alpha$ is a holomorphic isometry of $\mathbb{C H}^{2}$. Each geodesic ray is contained in the image of unique holomorphic totally-geodesic isometry $\phi: \mathbb{C H}^{1} \hookrightarrow$ $\mathbb{C H}^{2}$ satisfying $\gamma(t)=\phi(\tanh (t))$; in particular, $\phi_{1}(z)=(z, 0)$, for $z \in \Delta \cong \mathbb{C H}^{1}$. We note that every complex geodesic $\phi: \mathbb{C H}^{1} \hookrightarrow \mathbb{C H}^{2}$ arises uniquely (up to precomposition with an automorphism of $\mathbb{C H}^{1}$ ) as the intersection of the unit ball in $\mathbb{C}^{2}$ with a complex affine line.

Associated to each geodesic ray $\gamma:[0, \infty) \rightarrow \mathbb{C H}^{2}$ is a pair of transverse foliations of $\mathbb{C H}^{2}$, one by real geodesics asymptotic to $\gamma$ and another by complex hyperbolic horocycles asymptotic to $\gamma$. For each $p \in \mathbb{C H}^{2}$ there exists a unique geodesic $\gamma_{p}: \mathbb{R} \rightarrow$ $\mathbb{C H}^{2}$ and a unique time $t_{p} \in \mathbb{R}$ such that $\gamma_{p}\left(t_{p}\right)=p$ and $\lim _{t \rightarrow \infty} d_{\mathbb{C H}^{2}}\left(\gamma(t), \gamma_{p}(t)\right) \rightarrow 0$. For each $s \in \mathbb{R}_{+}$, we define the set $H(\gamma, s)=\left\{p \in \mathbb{C H}^{2} \mid \exp \left(t_{p}\right)=s\right\}$. The collection of subsets $\{H(\gamma, s)\}_{s \in \mathbb{R}_{+}}$defines the foliation of $\mathbb{C H}^{2}$ by complex hyperbolic horocycles asymptotic to $\gamma$.

Extremal length horocycles. Let $\gamma:[0, \infty) \rightarrow \mathcal{T}_{g, n}$ be a Teichmüller geodesic ray with unit speed. It has a unique lift to $\widetilde{\gamma}(t)=\left(X_{t}, q_{t}\right) \in Q_{1} \mathcal{I}_{g, n}$, such that $\gamma(t)=X_{t}$ and $\widetilde{\gamma}(t)=\operatorname{diag}\left(\mathrm{e}^{t}, \mathrm{e}^{-t}\right) \cdot\left(X_{0}, q_{0}\right)$. The map $q \mapsto(\mathcal{F}(q), \mathcal{F}(-q))$ gives an embedding $Q \mathcal{T}_{g, n} \hookrightarrow \mathcal{M} \mathcal{F}_{g, n} \times \mathcal{M F}_{g, n}$ which satisfies $\|q\|_{1}=i(\mathcal{F}(q), \mathcal{F}(-q))$ and 
sends the lift $\widetilde{\gamma}(t)=\left(X_{t}, q_{t}\right)$ of Teichmüller geodesic ray $\gamma$ to a path of the form $\left(\mathrm{e}^{t} \mathcal{F}(q), \mathrm{e}^{-t} \mathcal{F}(-q)\right)$.

The latter description of a Teichmüller geodesic and Theorem 2.1 show that the extremal length of $\mathcal{F}\left(q_{t}\right)$ along $\gamma$ satisfies $\lambda\left(\mathcal{F}\left(q_{t}\right), X_{s}\right)=\mathrm{e}^{2(t-s)}$ for all $t, s \in \mathbb{R}_{+}$, which motivates the following definition. For each $\mathcal{F} \in \mathcal{M} \mathcal{F}_{g, n}$ the extremal length horocycles asymptotic to $\mathcal{F}$ are the level-sets of extremal length $H(\mathcal{F}, s)=\{X \in$ $\left.\mathcal{T}_{g, n} \mid \lambda(\mathcal{F}, X)=s\right\}$ for $s \in \mathbb{R}_{+}$. The collection of subsets $\{H(\mathcal{F}, s)\}_{s \in \mathbb{R}_{+}}$defines the foliation of $\mathcal{T}_{g, n}$ by extremal length horocycles asymptotic to $\mathcal{F}$.

There is transverse foliation of $\mathcal{T}_{g, n}$ by real Teichmüller geodesics with lifts $\left(X_{t}, q_{t}\right)$ that satisfy $\mathcal{F}\left(q_{t}\right) \in \mathbb{R}_{+} \cdot \mathcal{F}$. One might expect that this foliation of $\mathcal{T}_{g, n}$ is analogous to the foliation of $\mathbb{C H}^{2}$ by geodesics that are positively asymptotic to $\gamma$. Although this is not always true, it is true for generic measured foliations $\mathcal{F} \in \mathcal{M} \mathcal{F}_{g, n}$.

Theorem 3.3 (H. Masur; [Mas80]). Let $\left(X_{t}, q_{t}\right)$ and $\left(Y_{t}, p_{t}\right)$ be two Teichmüller geodesics and $\mathcal{F}\left(q_{0}\right) \in \mathcal{M F}_{g, n}$ be uniquely ergodic. ${ }^{2}$ Then $\lim _{t \rightarrow \infty} d_{\mathcal{T}_{g, n}}\left(X_{t}, Y_{t}\right) \rightarrow 0$ if and only if $\mathcal{F}\left(q_{0}\right)=\mathcal{F}\left(p_{0}\right)$ in $\mathcal{M F}_{g, n}$ and $\lambda\left(\mathcal{F}\left(q_{0}\right), X_{0}\right)=\lambda\left(\mathcal{F}\left(p_{0}\right), Y_{0}\right)$.

REMARK. It is known that this result is not true for measured foliations that are not uniquely ergodic.

Proof of Theorem 3.1. Let $f: \mathbb{C H}^{2} \hookrightarrow \mathcal{T}_{g, n}$ be a holomorphic isometry for the Kobayashi metric. We summarize the proof in the following three steps:

1. Asymptotic behavior of geodesics determines the extremal length horocycles.

2. The geometry of horocycles determines the geometric intersection pairing.

3. Get a contradiction by a direct computation of the geometric intersection pairing.

Step 1. Let $X=f((0,0)) \in \mathcal{T}_{g, n}$ and $q, p \in Q_{1}(X)$ unit area quadratic differentials generating the two Teichmüller geodesic rays $f\left(\gamma_{1}\right), f\left(\gamma_{2}\right)$, where $\gamma_{1}, \gamma_{2}$ are two orthogonal geodesic rays in $\mathbb{C H} \mathbb{H}^{2}$ contained in the image of the totally real geodesic hyperbolic plane $\mathbb{R} \mathbb{H}^{2} \subset \mathbb{C H}^{2}$; explicitly, they are given by the formulas $\gamma_{1}(t)=(\tanh (t), 0), \gamma_{2}(t)=(0, \tanh (t))$, for $t \geq 0$.

For every $(X, q) \in Q_{1} \mathcal{T}_{q, n}$ there is a dense set of $\theta \in \mathbb{R} / 2 \pi \mathbb{Z}$ such that the measured foliation $\mathcal{F}\left(\mathrm{e}^{i \theta} q\right)$ is uniquely ergodic [KMS86]; hence, we can assume without loss of generality (up to a holomorphic automorphism of $\mathbb{C} \mathbb{H}^{2}$ ) that both $\mathcal{F}(q)$ and $\mathcal{F}(p)$ are (minimal) uniquely ergodic measured foliations. In particular, we can apply Theorem 3.3 to study the extremal length horocycles asymptotic to $\mathcal{F}(q)$ and $\mathcal{F}(p)$ respectively.

The complex hyperbolic horocycle $H\left(\gamma_{1}, 1\right)$ is characterized by the property that for the points $P \in H\left(\gamma_{1}, 1\right)$ the geodesic distance between $\gamma_{P}(t)$ and $\gamma_{1}(t)$ tends to zero as $t \rightarrow+\infty$, where $\gamma_{P}(t)$ is the unique geodesic with unit speed through $P$ that is positively asymptotic to $\gamma_{1}$. Applying Theorem 3.3 we conclude that:

$\overline{2}$ A measured foliation $\mathcal{F}$ is uniquely ergodic if it is minimal and admits a unique, up to scaling, transverse measure; in particular, $i(\gamma, \mathcal{F})>0$ for all simple closed curves $\gamma$. Compare with [Mas80]. 


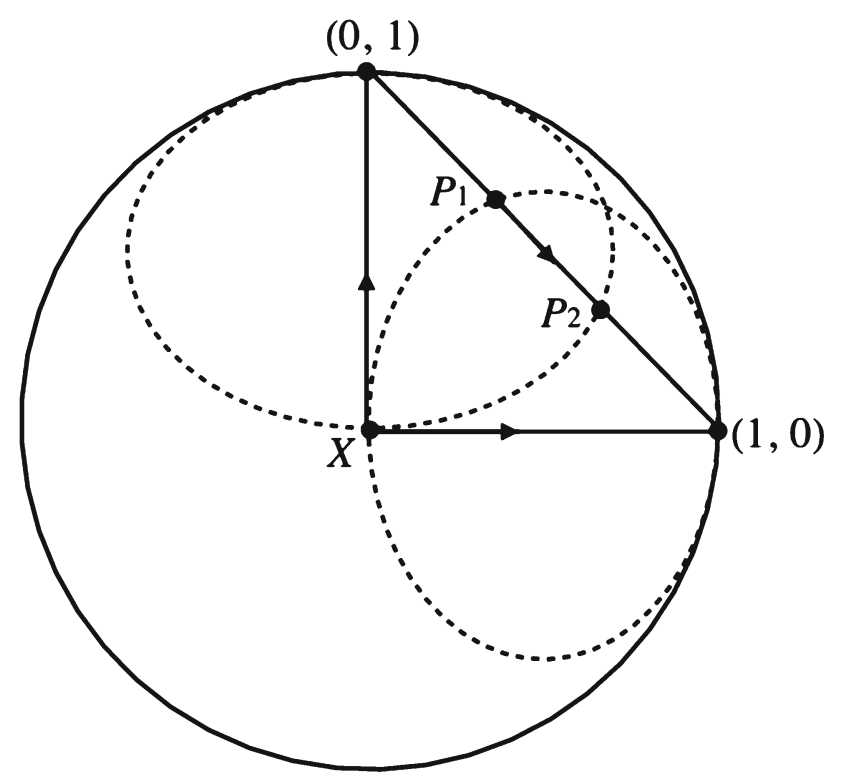

Figure 1: The real slice of $\mathbb{C H} \mathbb{H}^{2} \subset \mathbb{C}^{2}$ coincides with the Klein model $\mathbb{R} \mathbb{H}^{2} \subset \mathbb{R}^{2}$ of the real hyperbolic plane of constant curvature -1 .

$$
\begin{aligned}
& f\left(\mathbb{C H}^{2}\right) \cap H(\mathcal{F}(q), 1)=f\left(H\left(\gamma_{1}, 1\right)\right), \\
& f\left(\mathbb{C H}^{2}\right) \cap H(\mathcal{F}(p), 1)=f\left(H\left(\gamma_{2}, 1\right)\right) .
\end{aligned}
$$

Step 2. Let $\delta$ be the (unique) complete real geodesic in $\mathbb{C H}^{2}$, which is asymptotic to $\gamma_{1}$ in the positive direction and to $\gamma_{2}$ in the negative direction, i.e. its two endpoints are $(1,0),(0,1) \in \mathbb{C}^{2}$ in the boundary of the unit ball. Let $P_{1}$ and $P_{2}$ be the two points where $\delta$ intersects the horocycles $H\left(\gamma_{1}, 1\right)$ and $H\left(\gamma_{2}, 1\right)$, respectively. See 1 .

The image of $\delta$ under the map $f$ is a Teichmüller geodesic which is parametrized by a pair of measured foliations $\mathcal{F}, \mathcal{G} \in \mathcal{M F}_{g, n}$ with $i(\mathcal{F}, \mathcal{G})=1$ and its unique lift to $Q_{1} \mathcal{T}_{g, n}$ is given by $\left(\mathrm{e}^{t} \mathcal{F}, \mathrm{e}^{-t} \mathcal{G}\right)$, for $t \in \mathbb{R}$. Let $\widetilde{P}_{i}=\left(\mathrm{e}^{t_{i}} \mathcal{F}, \mathrm{e}^{-t_{i}} \mathcal{G}\right)$, for $i=1,2$, denote the lifts of $P_{1}, P_{2}$ along the geodesic $\delta$. Then, the distance between the two points is given by $d_{\mathbb{C H} \mathbb{H}^{2}}\left(P_{1}, P_{2}\right)=t_{2}-t_{1}$. From Step 1 , we conclude that $\mathrm{e}^{t_{1}} \mathcal{F}=\mathcal{F}(q)$ (3.1) and $\mathrm{e}^{-t_{2}} \mathcal{G}=\mathcal{F}(p)\left((3.2)\right.$. Therefore we have $i(\mathcal{F}(q), \mathcal{F}(p))=\mathrm{e}^{t_{1}-t_{2}}$.

REMARK. A simple calculation shows that $t_{2}-t_{1}=\log (2)$; hence, $i(\mathcal{F}(q), \mathcal{F}(p))=\frac{1}{2}$.

Step 3. The holomorphic automorphism given by $\phi(z, w)=\left(\mathrm{e}^{-i \theta} z, w\right)$, for $(z, w) \in$ $\mathbb{C} \mathbb{H}^{2}$, is an isometry of $\mathbb{C H}^{2}$ and sends the two horocycles $H\left(\gamma_{i}, 1\right)$ to the horocycles $H\left(\phi\left(\gamma_{i}\right), 1\right)$, for $i=1,2$. The Teichmüller geodesic ray $f\left(\phi\left(\gamma_{1}\right)\right)$ is now generated by $\mathrm{e}^{i \theta} q$, whereas the Teichmüller geodesic ray $f\left(\phi\left(\gamma_{2}\right)\right)$ is still generated by $p \in Q(X)$. Since the distance between $P_{1}$ and $P_{2}$ is equal to the distance between $\phi\left(P_{1}\right)$ and $\phi\left(P_{2}\right)$, using Step 2 and the continuity of the geometric intersection pairing we conclude that $i\left(\mathcal{F}\left(\mathrm{e}^{i \theta} q\right), \mathcal{G}\right)=\frac{1}{2}$ for all $\theta \in \mathbb{R} / 2 \pi \mathbb{Z}$. However, this contradicts the following Proposition 3.2. 
Proposition 3.2. Let $q \in Q_{1} \mathcal{T}_{g, n}$ and $\mathcal{G} \in \mathcal{M F}_{g, n}$. There exist $v_{1}, \ldots, v_{N} \in \mathbb{C}^{*}$ such that $i\left(\mathcal{F}\left(\mathrm{e}^{i \theta} q\right), \mathcal{G}\right)=\sum_{i=1}^{N}\left|\operatorname{Re}\left(\mathrm{e}^{i \theta / 2} v_{i}\right)\right|$ for all $\theta \in \mathbb{R} / 2 \pi \mathbb{Z}$.

Proof of Proposition 3.2. Let $q \in Q(X)$ be a unit area quadratic differential. We assume first that $q$ has no poles and that $\mathcal{G}$ is an isotopy class of simple closed curves. The metric given by $|q|$ is flat with conical singularities of negative curvature at its set of zeros and hence the isotopy class of simple closed curves $\mathcal{G}$ has a unique geodesic representative, which is a finite union of saddle connections of $q$. In particular, we can readily compute $i\left(\mathcal{F}\left(\mathrm{e}^{i \theta} q\right), \mathcal{G}\right)$ by integrating $\left|\operatorname{Re}\left(\sqrt{\mathrm{e}^{i \theta} q}\right)\right|$ along the union of these saddle connections. It follows that:

$$
i\left(\mathcal{F}\left(\mathrm{e}^{i \theta} q\right), \mathcal{G}\right)=\sum_{i=1}^{N}\left|\operatorname{Re}\left(\mathrm{e}^{i \theta / 2} v_{i}\right)\right| \quad \text { for all } \quad \theta \in \mathbb{R} / 2 \pi \mathbb{Z}
$$

where $N$ denotes the number of the saddles connections and $\left\{v_{i}\right\}_{i=1}^{N} \subset \mathbb{C}^{*}$ are their associated holonomy vectors.

We note that when $q$ has simple poles, there need not be a geodesic representative in $\mathcal{G}$ anymore. Nevertheless, Eq. (3.3) is still true by applying the argument to a sequence of length minimizing representatives.

Finally, we observe that the number of saddle connections $N$ is bounded from above by a constant that depends only on the topology of the surface. Combining this observation with the fact that any $\mathcal{G} \in \mathcal{M F}_{g, n}$ is a limit of simple closed curves and that the geometric intersection pairing $i(\cdot, \cdot): \mathcal{M F}_{g, n} \times \mathcal{M} \mathcal{F}_{g, n} \rightarrow \mathbb{R}$ is continuous, we conclude that Eq. (3.3) is true in general.

\section{Symmetric spaces versus Teichmüller spaces}

Let $\mathcal{T}_{g, n} \subset \mathbb{C}^{3 g-3+n}$ be a Teichmüller space and $\mathcal{B} \subset \mathbb{C}^{N}$ a bounded symmetric domain equipped with their Kobayashi metrics. In this section, we complete the proof of the following theorem.

Theorem 4.1. Let $\mathcal{B} \subset \mathbb{C}^{N}$ be a bounded symmetric domain and $\mathcal{T}_{g, n}$ be a Teichmüller space with $\operatorname{dim}_{\mathbb{C}} \mathcal{B}, \operatorname{dim}_{\mathbb{C}} \mathcal{T}_{g, n} \geq 2$. There are no holomorphic isometric immersions

$$
\mathcal{B} \stackrel{f}{\longleftrightarrow} \mathcal{T}_{g, n} \quad \text { or } \quad \mathcal{T}_{g, n} \stackrel{f}{\longrightarrow} \mathcal{B}
$$

equivalently, there are no holomorphic maps $f$ such that $d f$ is an isometry for the Kobayashi norms on tangent spaces.

REMARKs. 1. Torelli maps (associating to a marked Riemann surface the Jacobians of its finite covers) give rise to holomorphic maps $\mathcal{T}_{g, n} \stackrel{\mathcal{T}}{\longrightarrow} \mathcal{H}_{h}$ into bounded symmetric domains (Siegel spaces). It is known that these maps are not isometric for the Kobayashi metric in most directions. [McM]

2. For a similar result about holomorphic isometric submersions see [Ant].

Outline of the proofs. The proof that $\mathcal{B} \hookrightarrow \mathcal{T}_{g, n}$ follows from Theorem 3.1 (rank one) and a classical application of Sullivan's rigidity theorem (higher rank). The new 
ingredient we introduce in this section is a comparison of the roughness of Kobayashi metric for bounded symmetric domains and Teichmüller spaces, which we will use to prove that $\mathcal{T}_{g, n} \nrightarrow \mathcal{B}$

Preliminaries on symmetric spaces. We give a quick review of the main features of symmetric spaces, from a complex analysis perspective, which we use in the proof. We refer to [Hel78], [Sat80] for more details.

Let $\mathcal{B} \subset \mathbb{C}^{N}$ be a bounded symmetric domain and $p \in \mathcal{B}$. There is a unique, up to post-composition with a linear map, holomorphic embedding $\mathcal{B} \stackrel{i}{\longleftrightarrow} \mathbb{C}^{N}$ such that $i(\mathcal{B}) \subset \mathbb{C}^{N}$ is a strictly convex circular domain with $i(p)=0 \in \mathbb{C}^{N}$, which we refer to as the Harish-Chandra realization of $\mathcal{B}$ centered at $p \in \mathcal{B}$.

It is known that the Harish-Chandra realization of $\mathcal{B} \subset \mathbb{C}^{N}$ has the following useful description. There is a finite dimensional linear subspace $V_{\mathcal{B}} \subset M_{n, m}(\mathbb{C})$, of the space of complex $n \times m$ matrices, such that $\mathcal{B} \cong\left\{V \in V_{\mathcal{B}} \mid\|V\|_{\mathcal{B}}<1\right\}$ is the unit ball for the operator norm on $V_{\mathcal{B}}$, where $\|V\|_{\mathcal{B}}=\sup _{\|\xi\|_{2}=1}\|V(\xi)\|_{2}$, for $V \in M_{n, m}(\mathbb{C})$. We note that there is a natural identification $T_{p} \mathcal{B} \cong V_{\mathcal{B}} \cong \mathbb{C}^{N}$. [Sat80]

The Kobayashi norm on $T_{p} \mathcal{B} \cong V_{\mathcal{B}}$ coincides with the operator norm $\|V\|_{\mathcal{B}}$, for $V \in V_{\mathcal{B}} \subset M_{n, m}(\mathbb{C})$ and the Kobayashi distance from the origin is given by the formula $d_{\mathcal{B}}(0, V)=\frac{1}{2} \log \left(\frac{1+\|V\|_{\mathcal{B}}}{1-\|V\|_{\mathcal{B}}}\right)$, for $V \in \mathcal{B}$. [Kub89]

Roughness of the Kobayashi metric. The following proposition describes the roughness of the Kobayashi distance for bounded symmetric domains.

Proposition 4.2. Let $V:(-1,1) \rightarrow \mathcal{B}$ be a real-analytic path with $V(0) \neq p$. There is an integer $K>0$ and an $\epsilon>0$ such that $d_{\mathcal{B}}(p, V(\cdot)):[0, \epsilon) \rightarrow \mathcal{B}$ is a real-analytic function of $t^{1 / K}$ for $t \in[0, \epsilon)$.

Proof. Let $\mathcal{B}=\left\{\|V\|_{\mathcal{B}}<1\right\} \subset V_{\mathcal{B}} \subset M_{n, m}(\mathbb{C})$ be the Harish-Chandra realization of $\mathcal{B}$ centered at $p$. For each $t \in(-1,1)$, we denote by $\lambda_{i}(t)$, for $i=1, \ldots, n$, the eigenvalues of the (positive) square matrix $V(t)^{*} V(t)$, counted with multiplicities, where $V^{*}$ denotes the Hermitian adjoint of $V$.

The eigenvalues of $V(t)^{*} V(t)$ are the zeros of a polynomial, the coefficients of which are real-analytic functions of $t \in(-1,1)$. Therefore, the points $\left(t, \lambda_{i}(t)\right) \in \mathbb{C}^{2}$ for $i=1, \ldots, n$ and $t \in(-1,1)$ are contained in an algebraic curve $C=\{(t, \lambda) \in$ $\left.\mathbb{C}^{2} \mid P(t, \lambda)=0\right\}$, which is equipped with a finite-degree branched covering map to $\mathbb{C}$ given by $(t, \lambda) \mapsto t$, for $(t, \lambda) \in C$.

Since the operator norm is given by the formula $\|V(t)\|_{\mathcal{B}}=\sup \left\{\left|\lambda_{i}(t)\right|^{1 / 2}\right\}_{i=1}^{n}$, the proof of the proposition follows by considering the Puiseux series expansion for $\lambda_{i}(t)$ 's and the formula $d_{\mathcal{B}}(0, V(t))=\frac{1}{2} \log \left(\frac{1+\|V(t)\|_{\mathcal{B}}}{1-\|V(t)\|_{\mathcal{B}}}\right)$

The roughness of the Kobayashi metric for Teichmüller spaces is described by the following two theorems of M. Rees.

Theorem 4.3 (M. Rees; [Ree02]). The Teichmüller distance $d_{\mathcal{T}_{g, n}}: \mathcal{T}_{g, n} \times \mathcal{T}_{g, n} \rightarrow$ $\mathbb{R}_{\geq 0}$ is $C^{2}$-smooth on the complement of the diagonal $d_{\mathcal{T}_{g, n}}^{-1}(0)$. 
Theorem 4.4 (M. Rees; [Ree04]). When $\operatorname{dim}_{\mathbb{C}} \mathcal{T}_{g, n} \geq 2$, the Teichmüller distance $d_{\mathcal{T}_{g, n}}: \mathcal{T}_{g, n} \times \mathcal{T}_{g, n} \rightarrow \mathbb{R}_{\geq 0}$ is not $C^{2+\epsilon}$ for any $\epsilon>0$.

Moreover, let $X, Y \in \mathcal{T}_{g, n}$ be two distinct points connected by a (real) Teichmüller geodesic which is generated by a quadratic differential $q \in Q_{1}(X)$, with either a zero of order two or number of poles less than $n$. There is a real analytic path $X(t):(-1,1) \rightarrow \mathcal{T}_{g, n}$ with $X(0)=X$ such that the distance $d_{\mathcal{T}_{g, n}}(X(t), Y)$ is not $C^{2+h}$-smooth at $t=0$, for every gauge function $h(t)$ with $\lim _{t \rightarrow 0} \frac{h(t)}{1 / \log (1 /|t|)}=0$.

Proof of Theorem 4.1. Let $\mathcal{B} \subset \mathbb{C}^{N}$ be a bounded symmetric domain and $\mathcal{T}_{g, n}$ a Teichmüller space with $\operatorname{dim}_{\mathbb{C}} \mathcal{B}, \operatorname{dim}_{\mathbb{C}} \mathcal{T}_{g, n} \geq 2$. Using the fact that bounded symmetric domains and Teichmüller spaces contain holomorphic isometric copies of $\mathbb{C H}^{1}$ through every point and complex direction, and a theorem of Slodkowski [Sło91], [EM03], we deduce that any holomophic map $f$ between $\mathcal{B}$ and $\mathcal{T}_{g, n}$ which is an isometry for the Kobayashi norms on tangent spaces would be totally-geodesic and would therefore preserve the Kobayashi distance for pairs of points.

$\left(\mathcal{B} \leftrightarrow \mathcal{T}_{g, n}\right)$

Theorem 3.1 shows that there is no holomorphic isometry $f: \mathbb{C H}^{2} \rightarrow \mathcal{T}_{g, n}$. Moreover, an application of Sullivan's rigidity theorem (see [Tan93] for a precise statement) shows that there is no proper holomorphic map $f: \mathbb{C H}^{1} \times \mathbb{C H}^{1} \rightarrow \mathcal{T}_{g, n}$, hence neither is such a holomorphic map that is an isometry.

However, for every bounded symmetric domain $\mathcal{B}$ with $\operatorname{dim}_{\mathbb{C}} \mathcal{B} \geq 2$ there is either a holomorphic totally-geodesic isometry $\mathbb{C H}^{2} \hookrightarrow \mathcal{B}$ (rank one) or a holomorphic totally-geodesic isometry $\mathbb{C H}^{1} \times \mathbb{C H}^{1} \hookrightarrow \mathcal{B}$ (higher rank). [Kob70] We conclude that there is no holomorphic isometric immersion $f: \mathcal{B} \hookrightarrow \mathcal{T}_{g, n}$.

$\left(\mathcal{T}_{g, n} \nsucc \mathcal{B}\right)$

Let $f: \mathcal{T}_{g, n} \hookrightarrow \mathcal{B}$ be a holomorphic isometric immersion. Since $\operatorname{dim}_{\mathbb{C}} \mathcal{T}_{g, n} \geq 2$, we can choose two distinct points $X, Y \in \mathcal{T}_{g, n}$ as described in Theorem 4.4; hence there is a real analytic path $X(t):(-1,1) \rightarrow \mathcal{T}_{g, n}$ with $X(0)=X$ such that the Teichmüller distance $d_{\mathcal{T}_{g, n}}(X(t), Y)$ is not $C^{2+h}$-smooth at $t=0$ for every gauge function $h(t)$ with $\lim _{t \rightarrow 0} \frac{h(t)}{1 / \log (1 /|t|)}=0$.

Let $p=f(Y) \in \mathcal{B}$ and $V(\cdot):(-1,1) \rightarrow \mathcal{B}$ be the real analytic path given by $V(t)=f(X(t))$ for $t \in(-1,1)$. Theorem 4.3 shows $d_{\mathcal{B}}(p, V(t))$ is $C^{2}$-smooth at $t=0$ and Proposition 4.2 shows that it is real analytic in $t^{1 / K}$, for some fixed integer $K>0$, for all sufficiently small $t \geq 0$. Therefore, it follows that $d_{\mathcal{T}_{g, n}}(X(t), Y)$ is $C^{2+\frac{1}{K}}$-smooth, but this contradicts the choice of the path $X(t) \in \mathcal{T}_{g, n}$, given by Theorem 4.4, by considering the gauge function $h(t)=t^{1 / K}$ for $t \geq 0$. We conclude that there is no holomorphic isometric immersion $f: \mathcal{T}_{g, n} \hookrightarrow \mathcal{B}$.

\section{Acknowledgments}

I wish to thank my thesis advisor, Curtis T. McMullen, for many insightful discussions which set off the train of thought leading to the main result in this paper. 
Open Access This article is distributed under the terms of the Creative Commons Attribution 4.0 International License (http://creativecommons .org/licenses/by/4.0/), which permits unrestricted use, distribution, and reproduction in any medium, provided you give appropriate credit to the original author(s) and the source, provide a link to the Creative Commons license, and indicate if changes were made.

\section{References}

[Ant] S. M. Antonakoudis. A note on Teichmüller spaces and convex domains. In preparation.

[Ant] S. M. Antonakoudis. Royden's theorem and birational geometry. Preprint.

[Ber81] L. Bers. Finite dimensional Teichmüller spaces and generalizations. Bull. Amer. Math. Soc. 5(1981), 131-172.

[Bon88] F. Bonahon. The geometry of Teichmüller space via geodesic currents. Invent. math. 92(1988), 139-162.

[EKK94] C. J. Earle, I. Kra, and S. L. Krushkal. Holomorphic motions and Teichmüller spaces. Trans. Amer. Math. Soc. 343(1994), 927-948.

[EM03] C. J. EARLE and V. MARKovic. Isometries between the spaces of $L^{1}$ holomorphic quadratic differentials on Riemann surfaces of finite type. Duke Math. J. 120(2003), 433-440.

[FM12] B. FArb and D. Margalit. A Primer on Mapping Class Groups. Princeton University Press, 2012.

[FlP79] A. Fathi, F. Laudenbach, and V. PoÉnaru. Travaux de Thurston sur les surfaces. Astérisque, vol. 66-67, 1979.

[GL00] F. P. Gardiner and N. Lakic. Quasiconformal Teichmüller Theory. Amer. Math. Soc., 2000.

[Hel78] S. Helgason. Differential Geometry, Lie Groups, and Symmetric Spaces. Academic Press, 1978.

[HM79] J. Hubbard and H. Masur. Quadratic differentials and foliations. Acta Math. 142(1979), 221-274.

[Hub06] J. H. HubBard. Teichmüller Theory, vol. I. Matrix Editions, 2006.

[KMS86] S. Kerckhoff, H. Masur, and J. Smillie. Ergodicity of billiard flows and quadratic differentials. Ann. of Math. 124(1986), 293-311.

[Kob70] S. Kobayashi. Hyperbolic Manifolds and Holomorphic Mappings. Marcel Dekker, Inc., 1970.

[Kob98] S. Kobayashi. Hyperbolic Complex Spaces. Springer-Verlag, 1998.

[Kub89] Y. Kubota. On the Kobayashi and Carathéodory distances of bounded symmetric domains. Kodai Math. J. 12(1989), 41-48.

[Mas80] H. Masur. Uniquely ergodic quadratic differentials. Comment. Math. Helv. 55(1980), 255-266.

[McM03] C. McMullen. Billiards and Teichmüller curves on Hilbert modular surfaces. J. Amer. Math. Soc. 16(2003), 857-885.

[McM] C. McMullen. Entropy on Riemann surfaces and Jacobians of finite covers. Comment. Math. Helv., to appear.

[Ree02] M. REEs. Teichmüller distance for analytically finite surfaces is $C^{2}$. Proc. London Math. Soc.(3) 85(2002), 686-716. 
[Ree04] M. REes. Teichmüller distance is not $C^{2+\epsilon}$. Proc. London Math. Soc. (3) 88(2004), $114-134$.

[Roy71] H. L. Royden. Automorphisms and isometries of Teichmüller space. In Advances in the Theory of Riemann Surfaces, pages 369-384. Princeton University Press, 1971.

[Sat80] I. Satake. Algebraic Structures of Symmetric Domains. Princeton University Press, 1980.

[Sło91] Z. SŁodkowski. Holomorphic motions and polynomial hulls. Proc. Amer. Math. Soc. 111(1991), 347-355.

[Tan93] H. TAnigawA. Holomorphic mappings into Teichmüller spaces. Proc. Amer. Math. Soc. 117(1993), 71-78.

[Vee89] W. VeEch. Teichmüller curves in moduli space, Eisenstein series and an application to triangular billiards. Invent. math. 97(1989), 553-583.

Stergios M. Antonakoudis, Department of Pure Mathematics and Mathematical Statistics, Centre for Mathematical Sciences, University of Cambridge, Wilberforce Road,

Cambridge, CB3 0WB, UK

stergios@dpmms.cam.ac.uk

Received: September 6, 2016

Accepted: November 13, 2016 